\title{
Optical Properties of GaAs Nanowires with an Electric Potential That Varies Inversely with the Square of the Radial Distance
}

\author{
Moletlanyi Tshipa \\ Department of Physics, University of Botswana, Botswana \\ Correspondence should be addressed to Moletlanyi Tshipa; tshipam@mopipi.ub.bw
}

Received 18 January 2019; Revised 19 May 2019; Accepted 23 June 2019; Published 10 July 2019

Academic Editor: Yuri Galperin

Copyright (C) 2019 Moletlanyi Tshipa. This is an open access article distributed under the Creative Commons Attribution License, which permits unrestricted use, distribution, and reproduction in any medium, provided the original work is properly cited.

\begin{abstract}
A theoretical investigation of optical properties of a cylindrical quantum wire (CQW) is presented. The properties studied were optical absorption coefficient (AC) and change in refractive index (CRI) of the quantum wire. In particular, effect of an inverse parabolic potential on the optical properties of CQWs was investigated. This was done by solving the Schrödinger equation within the effective mass approximation to obtain the wave functions. The inverse parabolic potential reduces transition energies and therefore redshifts peaks of the $\mathrm{AC}$, as well as the anomalous dispersion region of the dependence of change in refractive index on the photon energy. The inverse parabolic potential also has effect on the magnitudes of these optical quantities, reducing the AC and enhancing the CRI. These properties of the inverse parabolic confining electric potential can have a wide range of applications in nanodevice technology, some details of which are discussed.
\end{abstract}

\section{Introduction}

The emergence of nanofabrication technology has ushered in an era in which quantum effects can be controlled for various applications and equally importantly, for probing natural phenomena on the nanoscale. Nanotechnology has enabled the scientific community to have at its disposal a plethora of nanostructures of various sizes and geometries [13]. These nanostructures have applications in a wide variety of disciplines. In medicine, they have been utilized in a broad spectrum of branches like osteology, for example, using a combination of nanowires and nanoparticles to repair bones with defects [4]. Silicon nanoribons have also been used as biosensors to detect carcinoembryonic antigen [5]. The potential of titanate nanowires to remove Uranium (VI) from the environment has been investigated [6]. Some nanowires have shown great potential to be used as photocatalysts as in the case of zinc oxide nanowires [7]. Wang et al. successfully demonstrated that double barrier nanostructures in conjunction with quantum dots can be used as photodetectors [8]. Other nanostructures have upconversion emission abilities which would be very useful in in vivo optical imaging [9], while others have been utilized to improve the efficiency of solar cells [10].
Most of these applications depend on optoelectronic properties of constituent nanostructures. It is thus vital to investigate optical properties of the constituent nanostructures. This has prompted researchers to turn their attention towards comprehending optical properties of nanostructures. Kumari et al. studied the dependence of change in refractive indices of nanostructures on both optical intensity and length of the nanowire [11]. Optical properties of laterally coupled quantum wires were studied, which revealed that the linear and nonlinear absorption coefficients become blueshifted when the nanowires get closer to each other [12]. Other studies on optical properties of nanowires have shown that external magnetic field and electric fields modify absorption coefficient of the nanostructures $[13,14]$. Effect of a tilted electric field on optical absorption of quantum wires has also been reported on [15]. The effect of noise on optical properties of impurity doped quantum dots has also been comprehensively studied [16-18]. Another way of manipulating optical properties is through spatially variant confining electric potentials, for example, the Tietz potential [19], potential steps [20], power exponential potential [21], and the inverse parabolic potential [22]. Spatially variant confining electric potentials are crucial as they can be used to modify properties of quantum 
structures without having to meddle with the sizes of the structures.

In this communication, the effect of an inverse parabolic potential on optical properties of nanowires is investigated. The inverse parabolic potential is superimposed on an infinite cylindrical square well (ICSW). The Schrödinger equation is solved for within the effective mass approximation. This report has the following organizational structure: Section 2 presents the theoretical treatment of the problem, Section 3 deals with results and discussions, and concluding remarks can be found in Section 4.

\section{Theoretical Model}

The envisaged system is a cylindrical quantum wire (CQW) of radius $R$ and very long length, which may be, for example, a gallium arsenide (GaAs) nanowire embedded in a glass matrix or may be a free standing cylindrical nanowire. The nanowire is envisioned to have a negatively charged strand (of radius $\rho_{0}$ ) lying along the axis of the nanowire, which through nanopatterning could be achieved by varying the lattice concentration. For $\rho_{0} \longrightarrow 0$, the confinement electric potential can be modelled as

$$
V(\rho)=\frac{1}{2} \mu \omega_{0}^{2} R^{2}\left(\frac{R^{2}}{\rho^{2}}-1\right) \quad(\rho<R)
$$

and infinity elsewhere, where $\mu$ is the effective mass of the electron and $\omega_{0}$ is the angular frequency associated with the classical harmonic oscillator. Considering the geometry of our system and due to the separability of the Hamiltonian, the wave function will be cast in the form $\psi(\rho, \phi, z)=C_{m l} \chi(\rho) \exp \left(i k_{z} z\right) \exp (i m \phi)$, in cylindrical coordinates. Here, $m$ is azimuthal quantum number which quantifies angular momentum, $l$ is radial quantum number, and $C_{m l}$ is normalization constant. $k_{z}$ is the axial wave number while $\chi(\rho)$ is the radial component of the wave function which satisfies the Schrödinger equation

$$
\begin{aligned}
& \frac{1}{\rho} \frac{d}{d \rho}\left(\rho \frac{\chi(\rho)}{d \rho}\right)+\left\{\frac{2 \mu}{\hbar^{2}}\left[E_{m l}-V(\rho)\right]-\frac{m^{2}}{\rho^{2}}\right\} \chi(\rho) \\
& \quad=0
\end{aligned}
$$

with $E_{m l}$ being the radial confinement energy. The solution to the above second-order differential equation is a linear combination of the Bessel $J$ and $Y$ functions. The Bessel $Y$ diverges at the origin and therefore has to be discarded as a solution for a solid cylindrical quantum wire, leaving the Bessel $J$ function being the only surviving solution:

$$
\chi(\rho)=J_{\nu l}(\kappa \rho) .
$$

Here, $\kappa=\sqrt{\mu\left(2 E_{m l}+\mu \omega_{0}^{2} R^{2}\right) / \hbar^{2}}$ and $\nu=\sqrt{m^{2}+\mu \omega_{0}^{2} R^{4} / \hbar^{2}}$. Applying the boundary condition at interfaces concerning the continuity of the wave function, one arrives at the expression for the energy spectrum of an electron confined in a CQW with an inverse parabolic potential as

$$
E_{T o t}=E_{m l}+E_{z}=\frac{\hbar^{2} j_{o m l}^{2}}{2 \mu R^{2}}-\frac{1}{2} \mu \omega_{0}^{2} R^{2}+\frac{\hbar^{2} k_{z}^{2}}{2 \mu}
$$

where $j_{\text {oml }}$ are the roots of the Bessel $J$ function and $E_{z}$ is the axial contribution to the total energy of the electron.

2.1. Optical Properties. Consider a circularly polarized monochromatic electromagnetic radiation of angular frequency $\omega$ incident on a cylindrical quantum wire. Absorption of the incident radiation is only possible if the energy of the radiation, $\hbar \omega$, is coincident with the energy difference between different states between which transitions are possible. Firstorder and third-order absorption coefficient of a crystal can be evaluated by utilizing the density matrix approach in conjunction with perturbation expansion method via [11-14]

$$
\begin{aligned}
& \alpha^{(1)}(\hbar \omega)=\frac{4 \pi \alpha_{F S} \sigma_{V}}{n_{c} e^{2}} \hbar \omega\left|M_{f i}\right|^{2} \delta\left(\Delta E_{f i}-\hbar \omega\right) \\
& \alpha^{(3)}\left(\hbar \omega, I_{\omega}\right) \\
& =-\frac{32 \pi^{2} \alpha_{F S}^{2} \sigma_{V} I_{\omega}}{n_{c}^{2} e^{4} \hbar \Gamma_{f i}} \hbar \omega\left|M_{f i}\right|^{4} \delta^{2}\left(\Delta E_{f i}-\hbar \omega\right)\left(1-\Xi_{\alpha}\right)
\end{aligned}
$$

where

$$
\begin{aligned}
\Xi_{\alpha} & =\frac{\left|M_{f f}-M_{i i}\right|^{2}}{4\left|M_{f i}\right|^{2}} \\
& \frac{\left(\hbar \omega-\Delta E_{f i}\right)^{2}-\left(\hbar \Gamma_{f i}\right)^{2}+2 \Delta E_{f i}\left(\Delta E_{f i}-\hbar \omega\right)}{\left(\Delta E_{f i}\right)^{2}+\left(\hbar \Gamma_{f i}\right)^{2}} .
\end{aligned}
$$

In the above, $\alpha_{F S}$ is the fine structure constant, $\sigma_{V}$ the electron volume density, and $n_{c}$ the refractive index of the crystal. $I_{\omega}$ is intensity of the incident radiation and $e$ is the electronic charge while $\Delta E_{f i}=E_{f}-E_{i}$ are transition energies, with $E_{i}$ and $E_{f}$ being energies of the initial $|i\rangle$ and final $|f\rangle$ states. $M_{f i}=\langle f|e \rho| i\rangle$ are the matrix elements coupling one state $|i\rangle$ to the other $|f\rangle$ while $\Gamma_{f i}$ are the linewidths associated with the different states indicated by the subscripts $i$ and $f$. The total $\mathrm{AC}$ is calculated according to

$$
\alpha\left(\hbar \omega, I_{\omega}\right)=\alpha^{(1)}(\hbar \omega)+\alpha^{(3)}\left(\hbar \omega, I_{\omega}\right)
$$

The energy conserving delta function has been replaced with the Lorentzian

$$
\delta\left(\Delta E_{f i}-\hbar \omega\right) \longrightarrow \frac{\hbar \Gamma_{f i}}{\pi\left[\left(\Delta E_{f i}-\hbar \omega\right)^{2}+\left(\hbar \Gamma_{f i}\right)^{2}\right]} .
$$

It is also useful to scrutinise the behaviour of refractive indices of quantum structures if they are to be effectively utilized in opto-electro-nanodevices. The first- and thirdorder contributions to the refractive index change are given by [13]

$$
\frac{\Delta n_{c}{ }^{(1)}(\hbar \omega)}{n_{c}}=\frac{\sigma_{V}}{2 \varepsilon_{c}}\left|M_{f i}\right|^{2} \frac{\Delta E_{f i}-\hbar \omega}{\left(\Delta E_{f i}-\hbar \omega\right)^{2}-\left(\hbar \Gamma_{f i}\right)^{2}}
$$




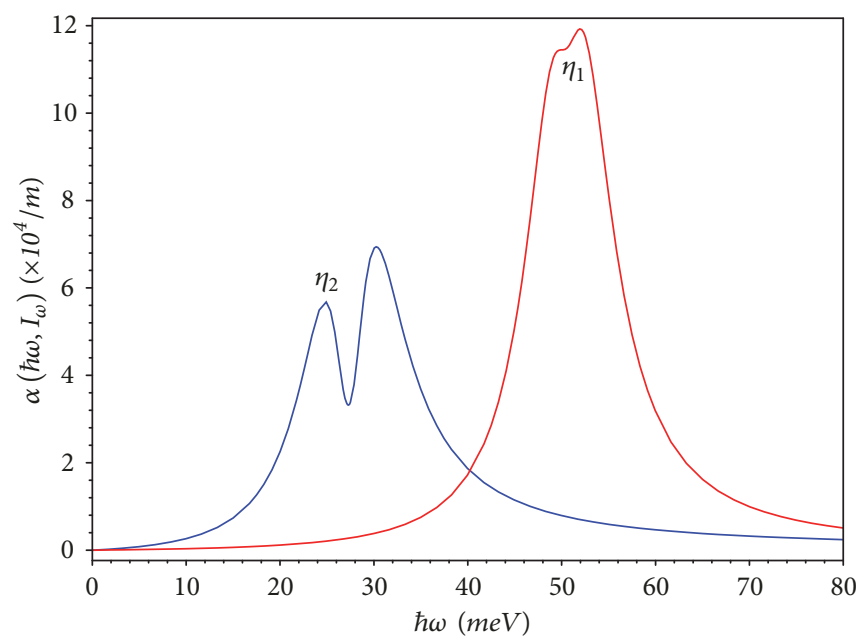

Figure 1: Dependence of the $(l=1)$ absorption coefficient, involving the ground state, on the incident photon energy for a CQW of radius $R=100 \AA$. The two graphs correspond to an ICSW $\left(\eta_{1}=\hbar \omega_{0}=0 \mathrm{meV}\right)$ and to $\eta_{2}=\hbar \omega_{0}=10 \mathrm{meV}$.

and

$$
\begin{aligned}
& \frac{\Delta n_{c}^{(3)}\left(\hbar \omega, I_{\omega}\right)}{n_{c}} \\
& =-\frac{\mu_{0} c I_{\omega}}{4 \varepsilon_{c} n_{c}^{2}} \frac{\sigma_{V}\left|M_{f i}\right|^{2}}{\left[\left(\Delta E_{f i}-\hbar \omega\right)^{2}-\left(\hbar \Gamma_{f i}\right)^{2}\right]^{2}} \Xi_{n}
\end{aligned}
$$

where

$$
\begin{aligned}
\Xi_{n} & =4\left|M_{f i}\right|^{2}\left(\Delta E_{f i}-\hbar \omega\right)-\left|M_{f f}-M_{i i}\right|^{2} \\
\cdot & \frac{\Delta E_{f i}\left(\Delta E_{f i}-\hbar \omega\right)^{2}-\left(\hbar \Gamma_{f i}\right)^{2}\left(3 \Delta E_{f i}-2 \hbar \omega\right)}{\left(\Delta E_{f i}\right)^{2}+\left(\hbar \Gamma_{f i}\right)^{2}} .
\end{aligned}
$$

In the above, $c$ is the speed of light in vacuum, $\varepsilon_{c}$ the permittivity of the CQW, and $\mu_{0}$ the permeability of the material. Similarly, the summed CRI is given by

$$
\frac{\Delta n}{n_{c}}=\frac{\Delta n_{c}^{(1)}(\hbar \omega)}{n_{c}}+\frac{\Delta n_{c}^{(3)}\left(\hbar \omega, I_{\omega}\right)}{n_{c}} .
$$

\section{Results and Discussion}

The values used in these computations are $0.067 m_{e}$ (relevant to GaAS crystals), $m_{e}$ being the mass of a free electron, $\sigma_{V}=$ $3 \times 10^{22} \mathrm{~m}^{-3}, n_{c}=3.2, \varepsilon_{c}=12.5$, and $\hbar \Gamma_{f i}=3.294 \mathrm{meV}$ [11]. Throughout this communication, the radius of the quantum wire has been held constant at $R=100 \AA$ and intensity of radiation fixed at $1200 \mathrm{MW} / \mathrm{m}^{2}$.

Figure 1 depicts the dependence of absorption coefficient on the photon energy for a quantum wire of radius $R=100 \AA$. The two graphs have been generated for an ICSW $\left(\eta_{1}=\right.$ $\hbar \omega_{0}=0 \mathrm{meV}$ ) and for a quantum wire with an inverse parabolic potential of strength $\eta_{2}=\hbar \omega_{0}=10 \mathrm{meV}$. Results for the $\hbar \omega_{0}=0 \mathrm{meV}$ case concur with those in the literature
$[14,23]$. As can be seen from the figure, the inverse parabolic potential redshifts peaks of the AC. This is due to the fact that this potential decreases transition energies as it increases in strength [24]. Transition energies are differences in the energies of states between which transitions occur. Also, the inverse parabolic potential diminishes the magnitude of the $\mathrm{AC}$, which is due to the fact that the potential closes the gap between the matrix elements $M_{i i}$ and $M_{f f}$; that is, $\left|M_{f f}-M_{i i}\right|$ decreases monotonically with increase in the inverse parabolic potential. The double peaks arise due to the (negative) third-order contribution of the absorption coefficient, and merge into a single peak for very low radiation intensities $\left(I_{\omega} \approx 0\right)$. Enhancement of the double peak in Figure 1 is due to the effect of the inverse parabolic potential on both the first- and the third-order contributions to the AC. This has been graphically illustrated in Figures 2(a) and 2(b), which depict the variation of the first (a) and third (Figure 2(b)) contributions to peaks of AC with the inverse parabolic potential. The three curves in Figures 2(a) and 2(b) have been generated for the first three radial quantum numbers in a cylindrical quantum wire of radius $R=100 \AA$, for the $m=0 \longrightarrow|m|=1$ transitions. First, this potential drastically reduces magnitudes of the peaks of first-order $\mathrm{AC}$ at resonance, regardless of the azimuthal and the radial quantum numbers involved. Second, peaks of the thirdorder AC initially decrease with increasing inverse parabolic potential strength and start increasing asymptotically after reaching a certain minimum for the $l=1$ transitions. For the $l>1$ cases, variation of the third-order AC is characterized by regions where the inverse parabolic potential decreases the magnitude of the third-order AC. For example, for the nanowire of radius $R=100 \AA$, this region is around $\hbar \omega_{0}=$ $82.6 \mathrm{meV}$ for $l=2$ and $\hbar \omega_{0}=35.1 \mathrm{meV}$ and $\hbar \omega_{0}=$ $231.5 \mathrm{meV}$ for $l=3$. Despite these slight variations, as the inverse parabolic potential increases, the third-order $\mathrm{AC}$ at resonance does not vary much, and for this case it is in the vicinity of $\sim-4 \times 10^{4} / \mathrm{m}$. This is in contrast to the first-order $\mathrm{AC}$ at resonance, which gets drastically reduced with increase 


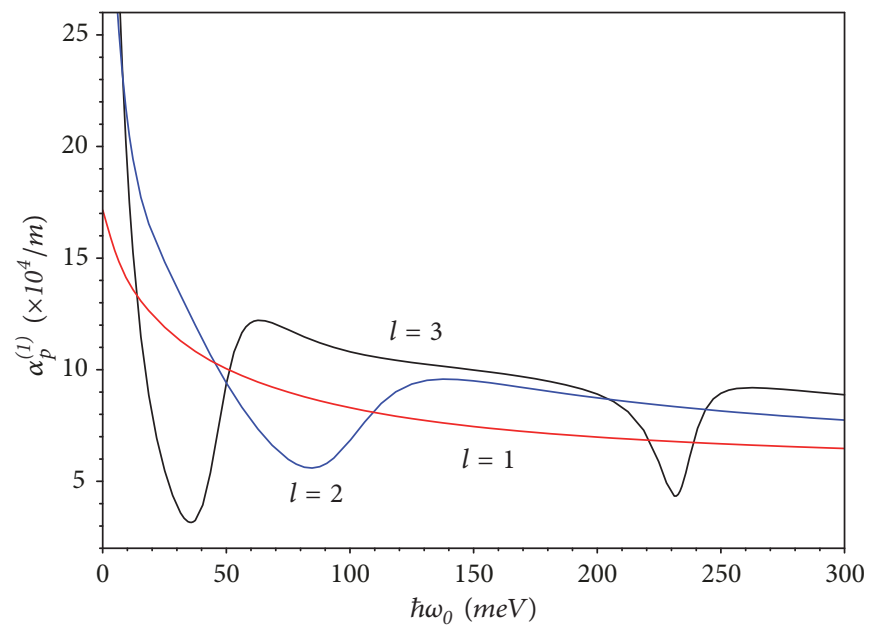

(a)

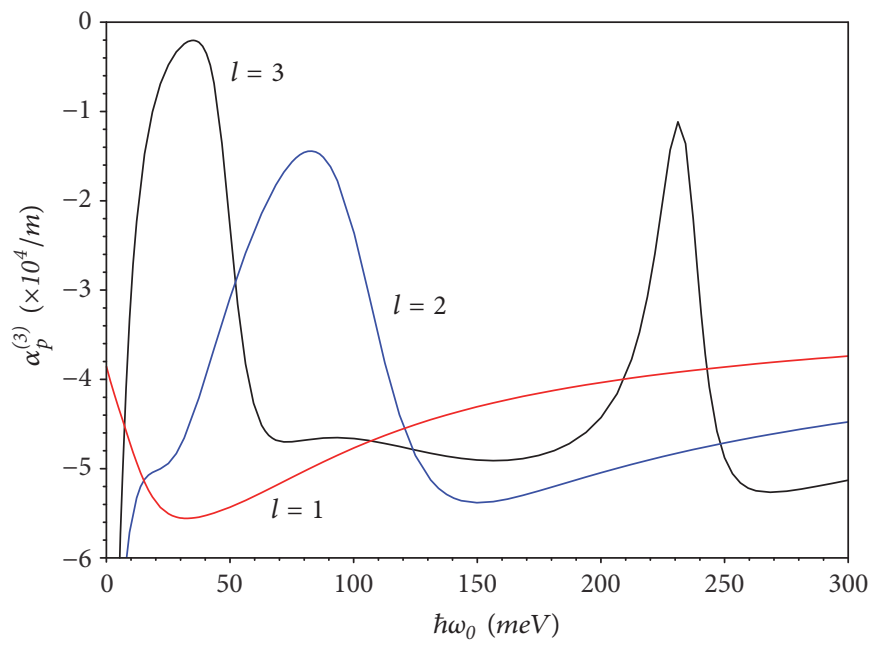

(b)

FIGURE 2: (a) Variation of first-order contribution to AC (at resonance), $\alpha_{p}^{(1)}$, with the inverse parabolic potential for a CQW of radius $R=$ $100 \AA$. The three curves have been generated for the first three radial quantum numbers $(l=1,2$ and 3$)$, for the $m=0 \longrightarrow|m|=1$ transition. (b) Same as for (a), but for the third-order contribution of AC, $\alpha_{p}^{(3)}$.

of the inverse parabolic potential. Thus, for the $l=1 \mathrm{AC}$, the inverse parabolic potential promotes the negative thirdorder contribution while it reduces the (positive) first-order contribution, consequently reducing $\mathrm{AC}$ at resonance. It is thus expected that the inverse parabolic potential will have reduced efficacy to enhance the development of double peaks for the $l>1$ transitions; for example in this case, potential strengths of $\hbar \omega_{0}=82.6 \mathrm{meV}$ (for $l=2$ ) and $\hbar \omega_{0}=35.1 \mathrm{meV}$ and $\hbar \omega_{0}=231.5 \mathrm{meV}$ (for $l=3$ ) will not promote the development of the double peaks.

Figure 3 shows the variation of AC with the inverse parabolic potential for a quantum wire of radius $R=100 \AA$, for the different values of the photon energy: $\omega_{1}=\hbar \omega=$ $20 \mathrm{meV}, \omega_{2}=\hbar \omega=25 \mathrm{meV}$, and $\omega_{3}=\hbar \omega=30 \mathrm{meV}$. Peaks of the AC shift to high values of the inverse parabolic potential as the photon energy decreases. Absorption occurs when the photon energy coincides with transition energies. For an infinite cylindrical square well (ICSW) $\left(\eta_{1}=\hbar \omega_{0}=0 \mathrm{meV}\right)$ of radius $=100 \AA$, transition energy between the ground state $(m=0)$ and the first excited state $(m=1)$ is around $\Delta E_{10}=50.6 \mathrm{meV}$. Thus, the value of the energy of radiation used in this figure is less than the $(m=0, m=1)$ transition energy. This means that electrons in an ICSW of this radius will neither absorb nor emit radiation having the selected energies in Figure 3 due to a transition, in this particular case, the $(m=0, m=1)$ transition. Since the inverse parabolic potential reduces transition energies, it can be used to tune nanowires in cases where the sizes of the nanowires are to have specific values. When photon energy decreases, higher values of the potential will be required to make the transition energies equal the photon energy, bringing the system into resonance.

Figure 4 illustrates AC (involving the ground state) as a function of strength of the inverse parabolic potential 


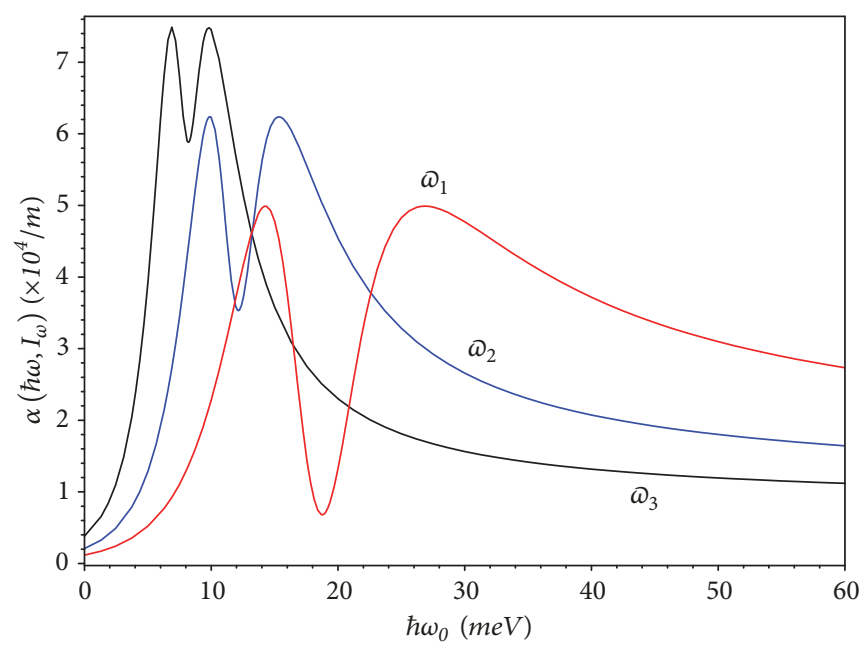

Figure 3: Variation of the $(l=1)$ AC (involving the ground state) with the inverse parabolic potential for a quantum wire of radius $R=100 \AA$ for the three photon energies, viz., $\omega_{1}=\hbar \omega=20 \mathrm{meV}, \omega_{2}=\hbar \omega=25 \mathrm{meV}$, and $\omega_{3}=\hbar \omega=30 \mathrm{meV}$.

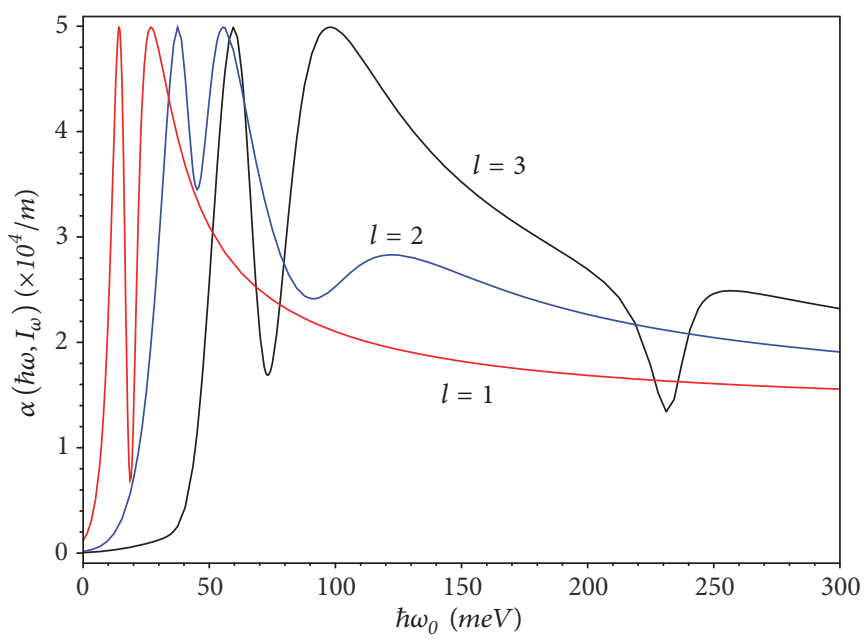

FIGURE 4: Dependence of AC (involving the ground state) on the inverse parabolic potential for three first three radial quantum numbers. The incident radiation has energy $\hbar \omega=20 \mathrm{meV}$ and radius of the quantum wire is $R=100 \AA$.

for the radial quantum numbers $l=1,2$ and $l=3$. Here, the quantum wire of radius $R=100 \AA$ is irradiated with an electromagnetic radiation of energy $\hbar \omega=20 \mathrm{meV}$. Transition energies associated with higher radial quantum numbers are generally larger than those associated with the lower radial quantum numbers. Consequently, for fixed photon energy, transitions of electrons with higher $l$ values require greater values of the inverse parabolic potential to bring the system to resonance.

Figure 5 depicts the scaled change in refractive index (CRI), $\Delta n / n_{c}$, of a cylindrical quantum wire of radius $R=$ $100 \AA$ as a function of the photon energy for different values of the inverse parabolic potential varying in steps of $2 \mathrm{meV}$ from $\hbar \omega_{0}=0 \mathrm{meV}$ (thick plot marked 0) to $\hbar \omega_{0}=30 \mathrm{meV}$ (plot marked 30). At low radiation field intensities, variation of the $\Delta n / n_{c}$ is characterized by regions where $\Delta n / n_{c}$ increases with increasing energy of the electromagnetic radiation (called the normal dispersion region) and a region where it decreases with increasing energy of the radiation (called the anomalous dispersion region) [25]. As already mentioned, the inverse parabolic potential decreases transition energies as it intensifies. This manifests as the redshifting of the CRI as the inverse parabolic potential increases. An additional effect of the inverse parabolic potential is enhancing the magnitude of the CRI. This is attributable to the fact that this potential "expels" electron away from the axis of the wire, signified by shifting of peaks of electron wave functions towards the walls of the nanowire [24]. This enhances the overlap between wave functions of the initial and final states.

Figure 6 illustrates the dependence the scaled CRI of a cylindrical quantum wire on the strength of the inverse parabolic potential for the first three radial quantum numbers. Here, electromagnetic radiation of energy $\hbar \omega=20 \mathrm{meV}$ is incident upon a cylindrical quantum wire of radius $R=$ $100 \AA$. Transition energies associated with greater radial 


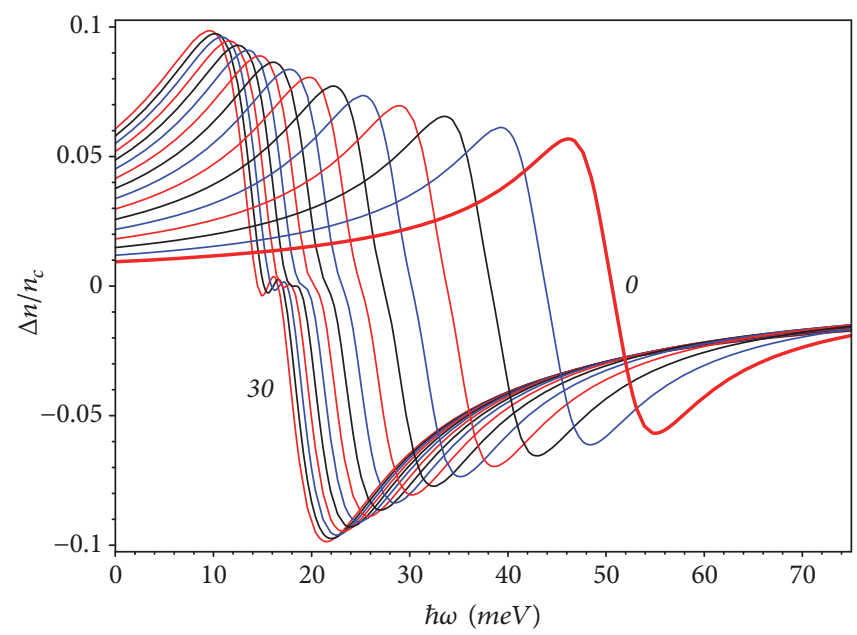

FIGURE 5: CRI as a function of the photon energy in a cylindrical nanowire of radius $R=100 \AA$ with an inverse parabolic potential of values from $\hbar \omega_{0}=0 \mathrm{meV}$ (thick plot marked 0 ) to $\hbar \omega_{0}=30 \mathrm{meV}$ (plot marked 30) in increments of $2 \mathrm{meV}$. Note the almost parabolic enhancement of the magnitude of maxima (and minima) of the CRI as the inverse parabolic potential increases in strength.

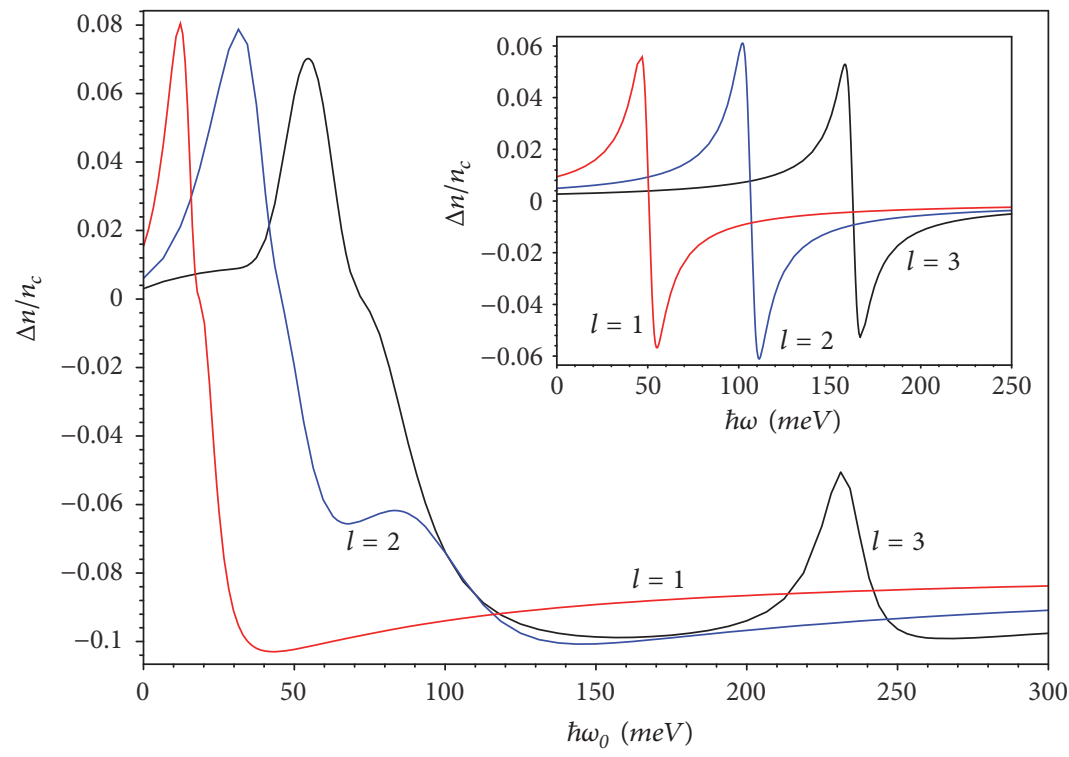

FIGURE 6: Scaled change in refractive index (CRI) of a CQW as a function of the inverse parabolic potential for the first three radial quantum numbers. The radius is $R=100 \AA$ and the photon energy remains fixed at $\hbar \omega=20 \mathrm{meV}$. The inset shows the scaled CRI of the cylindrical quantum wire as a function of the photon energy for the first three radial quantum numbers.

quantum numbers are usually greater than those associated with lower radial quantum numbers. This is also depicted in the inset of this figure, where the scaled CRI has been plotted as a function of photon energy for the first three radial quantum numbers: The inset shows that the anomalous dispersion region is shifted towards higher energy as the $l$ values increase. As such, for a given photon energy, higher values of the inverse parabolic potential are required to bring the system to resonance. Another feature of the dependence of the CRI on the inverse parabolic potential is the emergence of local peaks which are a consequence of the effect of this potential on the electron's radial wave function [24].

\section{Conclusions}

Optical properties of a cylindrical quantum wire with an inverse parabolic potential superimposed on an infinite cylindrical square well have been studied. The wave functions were obtained by solving the Schrödinger equation within the effective mass approximation. Results show that the inverse parabolic potential redshifts both the peaks of the absorption coefficient (AC) and the anomalous region of change in refractive index (CRI) of the cylindrical nanowire. Additionally, the inverse parabolic potential diminishes the magnitude of the AC while it enhances the magnitude of the CRI. These properties of the inverse parabolic potential make 
it a very attractive tool for manipulating optical properties of nanostructures, especially where the sizes the nanostructures are to have specific values. This can be very beneficial to the design of nanodevices.

\section{Data Availability}

The research was purely theoretical; no data were used.

\section{Disclosure}

The research and publication of this article was not funded by any financially supporting body.

\section{Conflicts of Interest}

The author declares that there are no conflicts of interest regarding the publication of this paper.

\section{Acknowledgments}

The author would like to express sincere gratitude to Lefika K. Maphage, Larona Radisigo, Othusitse E. Modise, and Modise Garegae for assisting with editing of the manuscript.

\section{References}

[1] S. Bae, H. Han, J. G. Bae et al., "Growth of silver nanowires from controlled silver chloride seeds and their application for fluorescence enhancement based on localized surface plasmon resonance," Small, vol. 13, no. 21, p. 1603392, 2017.

[2] T. Granz, S. Mariana, G. Hamdana et al., "Nanofabrication of vertically aligned 3D GaN nanowire arrays with sub-50 nm feature sizes using nanosphere lift-off lithography," Proceedings, vol. 1, no. 4, p. 309, 2017.

[3] Z. Khumalo, M. Blumenthal, M. Topić et al., "Oxide reduced silicon nanowires," Current Applied Physics, vol. 18, no. 5, pp. 576-582, 2018.

[4] T. Sun, W. Yu, Y. Zhu et al., "Porous nanocomposite comprising ultralong hydroxyapatite nanowires decorated with zinccontaining nanoparticles and chitosan: synthesis and application in bone defect repair," Chemistry - A European Journal, vol. 24, no. 35, pp. 8809-8821, 2018.

[5] Z. Bao, J. Sun, X. Zhao et al., “Top-down nanofabrication of silicon nanoribbon field effect transistor (Si-NR FET) for carcinoembryonic antigen detection," International Journal of Nanomedicine, vol. Volume 12, pp. 4623-4631, 2017.

[6] L. Yin, P. Wang, T. Wen et al., "Synthesis of layered titanate nanowires at low temperature and their application in efficient removal of U(VI)," Environmental Pollution, vol. 226, pp. 125134, 2017.

[7] Q. Zhou, J. Z. Wen, P. Zhao, and W. A. Anderson, "Synthesis of vertically-aligned zinc oxide nanowires and their application as a photocatalyst," Nanomaterials, vol. 7, no. 9, 2017.

[8] M. J. Wang, F. Y. Yue, and F. M. Guo, "Photoelectric characteristics of double barrier quantum dots-quantum well photodetector," Advances in Condensed Matter Physics, vol. 2015, Article ID 920805, 6 pages, 2015.

[9] L. Yin, T. Zeng, Z. Yi, C. Qian, and H. Liu, "Synthesis, tunable multicolor output, and high pure red upconversion emission of lanthanide-doped $\mathrm{Lu}_{2} \mathrm{O}_{3}$ nanosheets," Advances in Condensed Matter Physics, vol. 2013, Article ID 920369, 6 pages, 2013.

[10] Q. Qiu, P. Wang, L. Xu, D. Wang, Y. Lin, and T. Xie, "Photoelectrical properties of CdS/CdSe core/shell QDs modified anatase $\mathrm{TiO}_{2}$ nanowires and their application for solar cells," Physical Chemistry Chemical Physics, vol. 19, no. 24, pp. 15724-15733, 2017.

[11] P. Kumari, S. Sinha, and L. K. Mishra, "A theoretical evaluation of changes of refractive index as a function of photon energy for different incident optical intensities and fixed length of quantum wire," Journal of Pure Applied and Industrial Physics, vol. 7, no. 6, pp. 264-274, 2017.

[12] G. Liu, K. Guo, L. Xie, Z. Zhang, and L. Lu, "Tunability of linear and nonlinear optical absorption in laterally-coupled $\mathrm{Al}_{x} \mathrm{Ga}_{1-x} \mathrm{As} / \mathrm{GaAs}$ quantum wires," Journal of Alloys and Compounds, vol. 746, pp. 653-659, 2018.

[13] N. Arunachalam, A. John Peter, and C. K. Yoo, "Exciton optical absorption coefficients and refractive index changes in a strained InAs/GaAs quantum wire: The effect of the magnetic field," Journal of Luminescence, vol. 132, no. 6, pp. 1311-1317, 2012.

[14] M. Karimi and M. Hosseini, "Electric and magnetic field effects on the optical absorption of elliptical quantum wire," Superlattices and Microstructures, vol. 111, pp. 96-102, 2017.

[15] A. Bouazra, S. A. Nasrallah, and M. Said, "Intersubband optical absorption in InAs $/ \mathrm{In}_{0.52} \mathrm{Al}_{0.48}$ As quantum wire in the presence of tilted electric field," Optik - International Journal for Light and Electron Optics, vol. 147, pp. 328-333, 2017.

[16] J. Ganguly, S. Saha, S. Pal, and M. Ghosh, "Noise-driven optical absorption coefficients of impurity doped quantum dots," Physica E: Low-Dimensional Systems and Nanostructures, vol. 75, pp. 246-256, 2016.

[17] A. Mandal, S. Sarkar, A. P. Ghosh, and M. Ghosh, "Analyzing total optical absorption coefficient o impurity doped quantum dots in the presence of noise with special emphasis on electric field, magnetic field and confinement potential," Chemical Physics, vol. 463, pp. 149-158, 2015.

[18] S. Saha, S. Pal, J. Ganguly, and M. Ghosh, "Exploring optical refranctive index change of impurity doped quantum dots driven by white noise," Superlattices and Microstructures, vol. 88, pp. 620-633, 2015.

[19] R. Khordad and B. Mirhosseini, "Application of Tietz potential to study optical properties of spherical quantum dots," Pramana Journal of Physics, vol. 85, no. 4, pp. 723-737, 2015.

[20] S. Yilmaz and M. Şahin, “Third-order nonlinear absorption spectra of an impurity in a spherical quantum dot with different confining potential," Physica Status Solidi (b) - Basic Solid State Physics, vol. 247, no. 2, pp. 371-374, 2010.

[21] W. Xie, "Potential-shape effect on photoionization cross section of a donor in quantum dots," Superlattices and Microstructures, vol. 65, pp. 271-277, 2014.

[22] M. Tshipa, "Effect of an inverse parabolic confining electric potential on third harmonic generation in cylindrical quantum wires," Characterization and Application of Nanomaterials, vol. 1 , no. 3, 2018.

[23] M. Barseghyan, A. Kirakosyan, and D. Laroze, "Laser driven intraband optical transitions in two-dimensional quantum dots and quantum rings," Optics Communications, vol. 383, pp. 571576, 2017.

[24] M. Tshipa, "Oscillator strength for optical transitions in a cylindrical quantum wire with an inverse parabolic confining electric potential," Indian Journal of Physics, vol. 88, no. 8, pp. 849-853, 2014. 
[25] B. Akbarnavaz Farkoush, G. Safarpour, and A. Zamani, "Linear and nonlinear optical absorption coefficients and refractive index changes of a spherical quantum dot placed at the center of a cylindrical nano-wire: Effects of hydrostatic pressure and temperature," Superlattices and Microstructures, vol. 59, pp. 6676, 2013. 

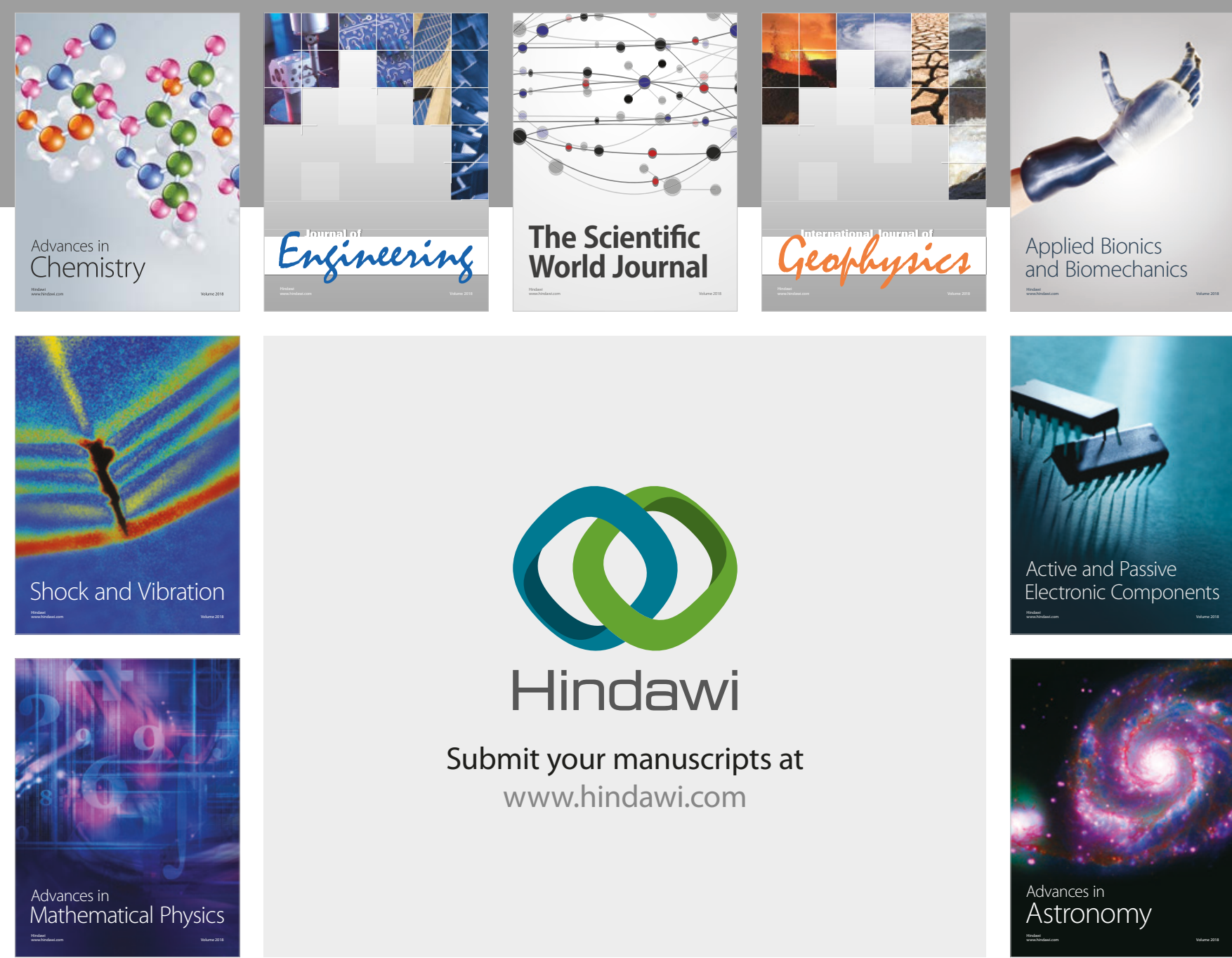

Submit your manuscripts at

www.hindawi.com

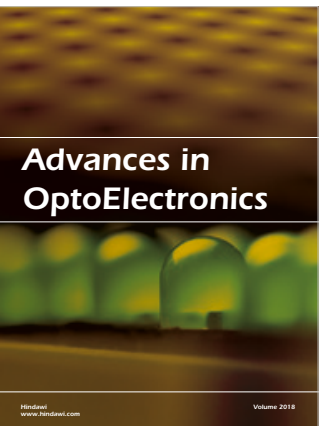

\section{Rotcting Machinery}
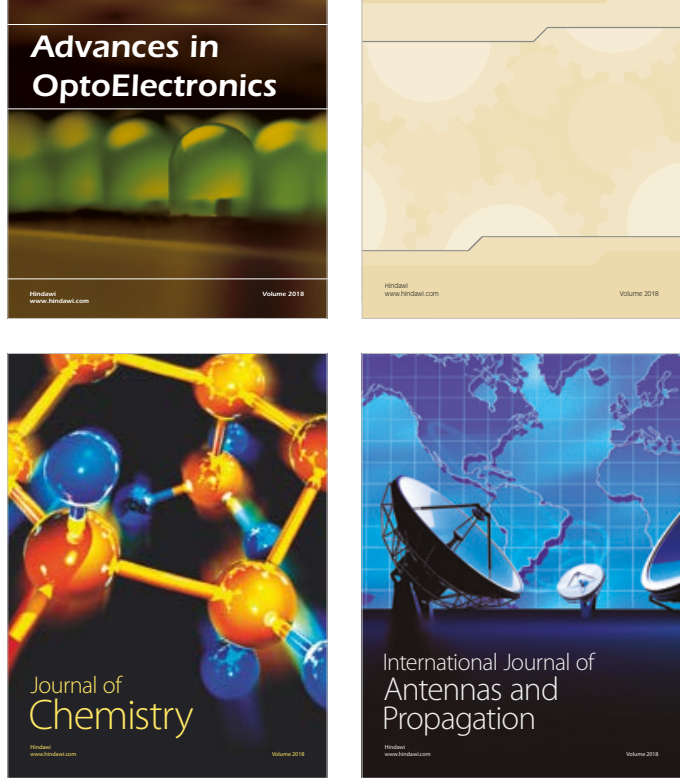

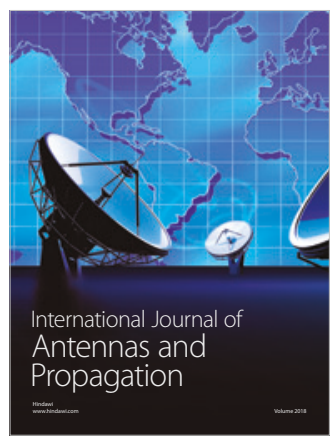

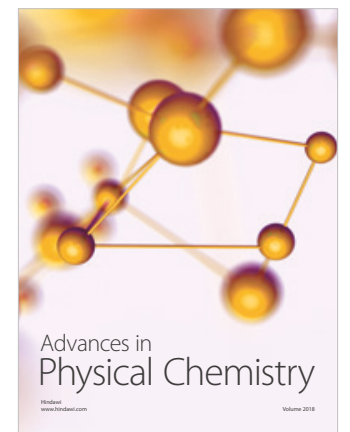

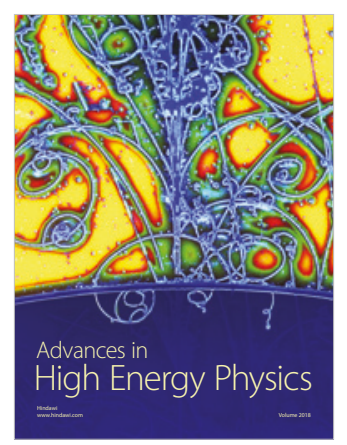

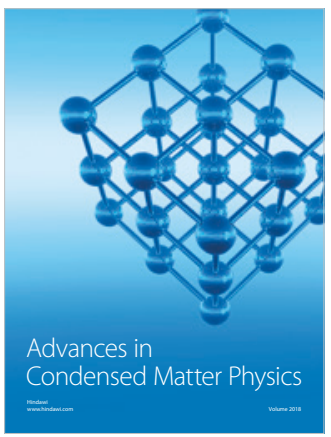

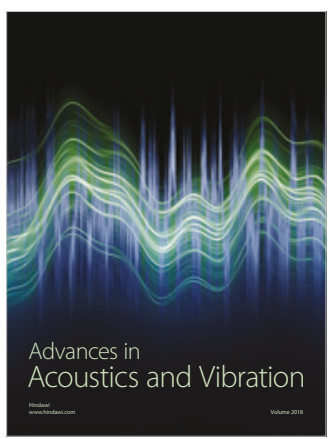

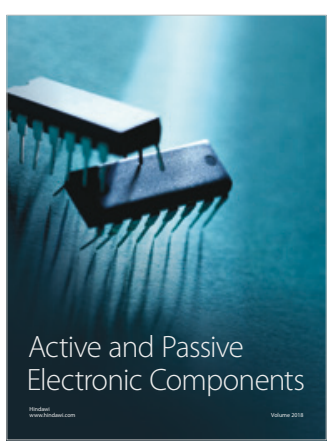
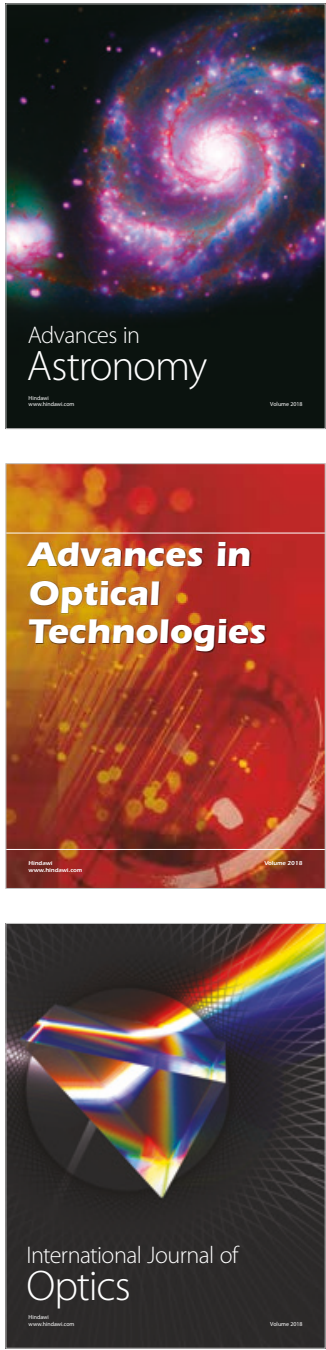